\title{
TROUBLESHOOTING PLANTWIDE OSCILLATIONS USING NONLINEARITY INFORMATION
}

\author{
M. A. A. Shoukat Choudhury \\ Department of Chemical Engineering \\ Bangladesh University of Engineering and Technology
}

\begin{abstract}
Plant-wide oscillations are common in many processes. Their effects propagate to many units and may impact the overall process performance. It is important to detect and diagnose the cause of such oscillations in order to rectify the situation and maintain the proper profitability of the plant. This paper proposes a new procedure to detect and diagnose plant-wide oscillations using routine operating data. The method has been developed based on the nonlinearity information in the process data. A new Total Nonlinearity Index (TNLI) has been defined to quantify nonlinearities. The method is based on the assumption that the nonlinearity is highest near the source and decreases as one moves away from the source. This assumption is true because chemical processes have the nature of low-pass filters and they filter out gradually higher order harmonics of the signals. Signals with higher order harmonics are generally more nonlinear. The proposed diagnostic method has already been successfully applied for troubleshooting many industrial plant-wide oscillation problems. Two of such case studies have been presented in this paper.
\end{abstract}

\section{Introduction}

Oscillations are a common form of plant-wide disturbance. The presence of oscillations in control loops increases the variability of the process variables thus causing inferior quality products, larger rejection rates, increased energy consumption and reduced average throughput. The high level of energy integration in most modern plants facilitates the propagation of oscillations from one process unit to another. It is important to detect and diagnose the causes of oscillations in a chemical process because a plant running close to product quality limits or operating constraints is more profitable than a plant that has to back away due to the amplitude of the oscillations (Martin et al., 1991; Shunta, 1995). In this paper, we present a new method for the detection and diagnosis of plant-wide oscillations and demonstrate its efficacy through an application to a chemical process at Eastman Chemical Company, USA.

In a control loop, oscillations may arise for various reasons including poorly tuned controllers, presence of oscillatory disturbances and nonlinearities. Bialkowski (1992) reported that about $30 \%$ of the loops are oscillatory due to control valve problems. In a recent industrial survey, Desborough and Miller (2002) found that control valve problems account for about a third of the $32 \%$ of controllers classified as "poor" or "fair".

The method presented here is quite general for finding the root cause of oscillations due to nonlinearities, which is known to be the key reason for oscillations in process industries (Bialkowski, 1992; Desborough and
Miller, 2002). The attraction of this method is that it primarily uses only regular operating data. No additional experimentation of the plant is required. The main contributions of this paper are:

(1) Methods for automatic detection of multiple oscillations across an entire plant site.

(2) Development of new indices based on higher order statistics for diagnosing root cause of plant-wide oscillations.

(3) Demonstration of the potential of systematic use of signal processing techniques for solving industrial problems through case studies.

\section{What is Plantwide Oscillation(s)}

When one or more oscillations is generated somewhere in the plant and propagates throughout a whole plant or some units of the plant, such oscillations are termed as plantwide or unitwide oscillation. Oscillation may propagate through many units of the process plants because of the tight heat and mass integration in the plant as well as the presence of recycle streams in the plant. Figure 1 shows an example of a plant-wide oscillation problem. The left panel shows the time trends of 37 variables representing a plant-wide oscillation problem in a refinery (courtesy of SouthEast Asia Refinery). The right panel shows the power spectrum of these variables. This clearly shows the presence of an oscillation with a frequency of 0.06 or approximately 17 samples/cycle in many of these variables. The presence of such plant-wide oscillation(s) take a huge toll on the plant economy. 


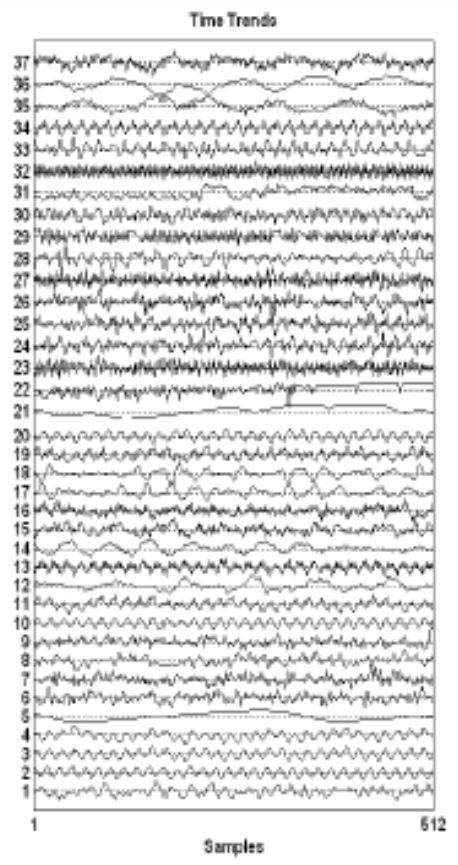

(a) Time Trends

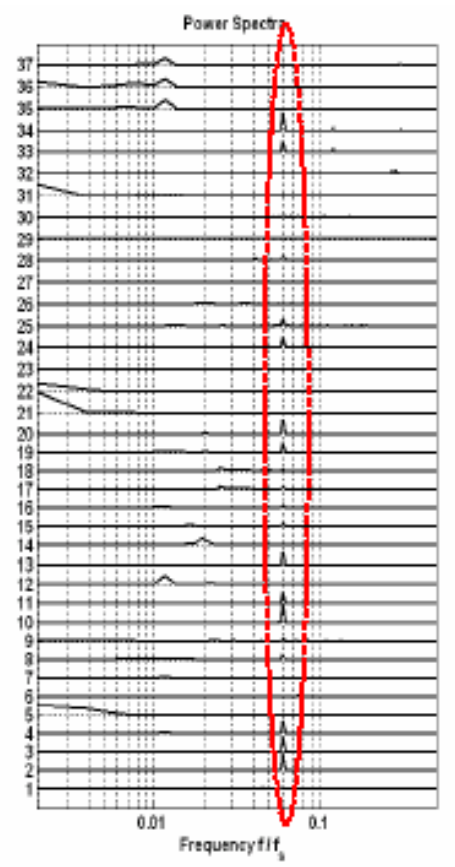

(b) Power Spectra

Fig 1: Time trends and their power Spectra

\section{Detection of Plantwide Oscillations}

Detection of plantwide oscillation may not be a difficult problem. Often times the plant operators notice some oscillations in the plant, which leads to a deeper investigation of the problem and may cause the invention of a plantwide oscillation of a larger nature. However, it is difficult to find all variables oscillating together out of the hundreds or thousands of variables in a chemical plant. Before a full scale oscillation diagnosis exercise is undertaken, it is important to find all signals oscillating at the same frequency as the root cause generally lies within this set. In contrast to this, most of the available techniques for detection focus on a loop by loop analysis (Hagglund, 1995; Taha et al., 1996; Miao and Seborg, 1999). Recently, Thornhill and co-workers have presented some detection tools that consider the plant-wide nature of oscillations (Thornhill et al., 2003a). To detect oscillations in process measurements and identify signals with common oscillatory behavior, use of spectral principal component analysis (Thornhill et al., 2002) or autocorrelation functions (acf) (Thornhill et al., 2003a) is suggested. Over the last few years, some studies were carried out to detect plant-wide oscillations (Tangirala et al., 2005; Jiang et al., 2006) and to group the similar oscillations together. The following are the brief description of some the techniques that can be used for detecting plant-wide oscillation(s).

\subsection{High Density Plot - An excellent Visualisation Tool}

This plot describes time series data and their spectra in a nice compact form in one plot. From this plot, one can easily visualize the nature of the data and the presence of common oscillation(s) in the data. However, this method cannot provide a list of the variables that oscillate together automatically. Figure 1 is an example of a high density plot.

\subsection{Power Spectral Correlation Map (PSCMAP)}

The power spectral correlation index (PSCI) is defined as the correlation between the power spectra of two different measurements. It is a measure of the similarity of spectral shapes, i.e., measure of the commonness of frequencies of oscillations.

The Discrete Fourier Transforms (DFT) that are used to calculate the spectrum are calculated after removal of means from the time-series data. However, the correlation used in the calculation of PSCI is calculated without the removal of mean of the spectra. The PSCI for any two spectra $\left|X_{i}(\omega)\right|^{2}$ and $|X j(\omega)|^{2}$ is calculated as

$\operatorname{correlation}\left(\left|X_{i}(\omega)\right|^{2},\left|X_{j}(\omega)\right|^{2}\right)=\frac{\sum_{\omega_{k}}\left|X_{i}\left(\omega_{k}\right)\right|^{2}\left|X_{j}\left(\omega_{k}\right)\right|^{2}}{\sqrt{\left|X_{i}\left(\omega_{k}\right)\right|^{4}\left|X_{j}\left(\omega_{k}\right)\right|^{4}}}$ 
As a result, PSCI always lies between 0 and 1 . The phase information is excluded due to taking the magnitude of the DFT of the measurements. In the detection of plant-wide oscillations, the objective is to collect variables with similar oscillatory behaviour. For multivariate processes, the PSCI is a matrix of size $m \times m$, where $m$ is the number of measured variables.

In order to provide an effective interpretation of the PSCI, the matrix is plotted as a colour map, which is termed as the power spectral correlation map. The intensity as well as the type of colour in the map is assigned in proportion to the value of the correlation index. This mapping is performed according to the choice of colour and the number of shades in that colour. An important aspect of this colour map is its ability to automatically re-arrange and group variables together with similar shapes, i.e., variables, which oscillate at a common frequency and have therefore similar values of PSCI. For a detailed discussion on this method, refer to (Tangirala et al., 2005). This method provides a list of the variables that are oscillating together.

\section{Diagnosis Techniques for Plant-wide Oscillations}

As described in the last section, the detection of plantwide oscillation is relatively an easy problem compared to the diagnosis of its root-cause. Recently there appeared a few papers that described a few techniques to perform root-cause diagnosis of plantwide oscillation (Zang and Howell, 2006; Zang and Howell, 2005; Thornhill et al., 2003b; Thornhill et al., 2001).

Industrial processes generally deviate from Gaussianity and linearity and exhibit nonlinear behaviour. The distribution of signals from nonlinear processes is often skewed and non-normal. These processes can conveniently be studied using Higher Order Statistics (HOS) (Nikias and Petropulu, 1993). Among the various frequency domain HOS measures the bispectrum is the simplest. It is the frequency domain counterpart of the third order moments and defined as

$$
B\left(f_{1}, f_{2}\right) \triangleq E\left[X\left(f_{1}\right) X\left(f_{2}\right) X^{*}\left(f_{1}+f_{2}\right)\right]
$$

where, $X(f)$ is the Fourier transformation of the data series $x(t)$. Equation 2 shows that it is a complex quantity having both magnitude and phase. It can be plotted against two independent frequency variables, $f_{1}$ and $f_{2}$ in a three dimensional plot. Each point in the plot represents the bispectral energy content of the signal at the bifrequency, $\left(f_{1}, f_{2}\right)$. In fact, the bispectrum, $B\left(f_{1}, f_{2}\right)$, at point $\left(f_{1}, f_{2}\right)$ measures the nonlinear interaction between frequencies $f_{1}$ and $f_{2}$ (Nikias and Petropulu, 1993). This interaction between frequencies can be related to the nonlinearities present in the signal generating systems and therein lies the core of its usefulness in the detection and diagnosis of nonlinearities.

The bispectrum is normalized in the following way to give a measure called bicoherence whose magnitude is bounded between 0 and 1 :

$$
b i c^{2}\left(f_{1}, f_{2}\right) \triangleq \frac{\left|E\left[B\left(f_{1}, f_{2}\right)\right]\right|^{2}}{E\left[\left|X\left(f_{1}\right) X\left(f_{2}\right)\right|^{2}\right] E\left[\left|X\left(f_{1}+f_{2}\right)\right|^{2}\right]}
$$

where 'bic' is known as 'bicoherence' function. Equation 3 can be rewritten as

$$
b i c^{2}\left(f_{1}, f_{2}\right) \triangleq \frac{\left|E\left[X\left(f_{1}\right) X\left(f_{2}\right) X^{*}\left(f_{1}+f_{2}\right)\right]\right|^{2}}{E\left[\left|X\left(f_{1}\right) X\left(f_{2}\right)\right|^{2}\right] E\left[\left|X\left(f_{1}+f_{2}\right)\right|^{2}\right]}
$$

If Welch's periodogram method is used to estimate the bicoherence, the expectation operator can be replaced with a summation operator over the number of data segments using the assumption of ergodicity:

$$
\operatorname{bic}^{2}\left(f_{1}, f_{2}\right)=\frac{\left|\frac{1}{M} \sum_{i=1}^{M} X_{i}\left(f_{1}\right) X_{i}\left(f_{2}\right) X_{i}^{*}\left(f_{1}+f_{2}\right)\right|^{2}}{\frac{1}{M} \sum_{i=1}^{M}\left|X_{i}\left(f_{1}\right) X_{i}\left(f_{2}\right)\right|^{2} \cdot \frac{1}{M} \sum_{i=1}^{M}\left|X_{i}\left(f_{1}+f_{2}\right)\right|^{2}}
$$

The squared bicoherence is usually estimated using this equation.

\subsection{Test of Gaussianity and Linearity of a Signal}

Two indices - Non-Gaussianity Index (NGI) and nonlinearity Index $(N L I)$ - were developed in Choudhury et al. (2004) to test the Gaussianity and linearity of a signal or time series. The indices were developed based on the statistical hypothesis test on the average squared bicoherence in the principal domain $\left(0<f_{1}<0.5, f_{2}<f_{1}\right.$, and $\left.2 f_{1}+f_{2}<1\right)$ of the bicoherence. The average bicohernce was used in the formulation of the indices because the estimation of bicoherence was not free from spurious peaks. The new estimation method described in (Choudhury et al., $2006 a$ ) allows us to modify the previously published indices. The modified indices have fewer cases of false positives and better capability of detecting nonlinearities. The following section describes the modification of the indices with a brief overview of the old indices. 
A discrete ergodic stationary time series, $x(k)$, is called linear if it can be represented by

$$
x(k)=\sum_{n=0}^{N-1} h(n) e(k-n)
$$

where $e(k)$ is a sequence of independent identically distributed random variables with $E[e(k)]=0, \sigma^{2}=$ $E\left[e^{2}(k)\right]$, and $\mu_{3}=E\left[e^{3}(k)\right]$. For a linear time series, it can be shown that

$$
\operatorname{bic}^{2}\left(f_{1}, f_{2}\right)=\frac{\mu_{3}^{2}}{\sigma_{e}^{6}}
$$

Equation 7 shows that for any linear signal, $x$, the squared bicoherence will be independent of the bifrequencies, i.e., a constant in the bifrequency plane. If the squared bicoherence is zero, the signal $x$ is Gaussian because the skewness or $\mu_{3}$ is also zero in such a case. To check whether the squared bicoherence is constant, two tests are required. One is to determine whether the squared bicoherence is zero, which would show that the signal is Gaussian and thereby the signal generating process is linear. The other is to test for a non-zero constant value of the squared bicoherence which would show that the signal is non-Gaussian but the signal generating process is linear.

\subsubsection{Modified Non-Gaussianity Index (NGI $\left.I_{\text {modified }}\right)$}

It is well established in the HOS literature that bicoherence is a complex normal variable, i.e., both estimates of real and imaginary parts of the bicoherence are normally distributed (Hinich, 1982) and asymptotically independent, i.e., the estimate at a particular bifrequency is independent of the estimates of its neighboring bifrequencies. Therefore, the squared bicoherence at each bifrequency is a noncentral chi-squared $\left(\chi^{2}(m)\right)$ distributed variable with 2 degrees of freedom and mean, $m$.

The following statistical test can be applied to check for the significance of bicoherence magnitude at each individual bifrequency:

$$
P\left\{2 K \operatorname{bic}^{2}\left(f_{1}, f_{2}\right)>c_{\alpha}^{\chi^{2}}\right\}=\alpha
$$

or,

$$
P\left\{\operatorname{bic}^{2}\left(f_{1}, f_{2}\right)>\frac{c_{\alpha}^{\alpha^{2}}}{2 K}\right\}=\alpha
$$

where $K$ is the number of data segments used in bicoherence estimation, $c_{\alpha}^{\chi^{2}}$ is the critical value calculated from the central $\chi^{2}$ distribution table for a significance level of $\alpha$ at degrees of freedom 2. For example, at $\alpha=0.05$, the value of $c_{0.05}^{\chi^{2}}$ is 5.99 .

Often the principal domain of the bicoherence plot contains more than a hundred bifrequencies. The hypothesis test results for this large number of bifrequencies can be conveniently summarized into the following modified Non-Gaussianity Index ( $\left.N G I_{\text {modified }}\right)$

$$
N G I_{\text {modified }} \triangleq \frac{\sum b i c_{\text {gignificant }}^{2}}{L}-\frac{c_{\alpha}^{\chi^{2}}}{2 K}
$$

where $b i c^{2}$ significant are those bicoherence which fail the hypothesis test in Equation (9), i.e., $\operatorname{bic}^{2}\left(f_{1}, f_{2}\right)>\frac{c_{\alpha}^{\chi^{2}}}{2 \mathrm{~K}}$, and $L$ is the number of $b i c^{2}$ significant. Therefore, the following rule-based decision is suggested:

- if $N G I_{\text {modified }} \leq \alpha$ the signal is GAUSSIAN

- $\quad$ if $N G I_{\text {modified }}>\alpha$, the signal is NON-GAUSSIAN

Thus, a signal is non-skewed or Gaussian at a confidence level of $\alpha$ if the $N G I_{\text {modified }}$ is less than or equal to zero. This index has been defined to facilitate the automation of this decision.

\subsubsection{Modified Nonlinearity Index (NLI $\left.I_{\text {modified }}\right)$}

If a signal is found to be Gaussian, the signal generating process is assumed to be linear (Rao and Gabr, 1980). In the case of a non-Gaussian signal, the signal generating process should be tested for its linearity. As shown in Equation 7, if the signal is nonGaussian and linear, the magnitude of the squared bicoherence should be a non-zero constant at all bifrequencies in the principal domain because $\mu$ is a non-zero constant.

A simple way to confirm the constancy of squared bicoherence is to have a look at the 3-D squared bicoherence plot and observe the flatness of the plot. If the squared bicoherence is of a constant magnitude at all bifrequencies in the principal domain, the variance of the estimated bicoherence should be zero. To check the flatness of the plot or the constancy of the squared bicoherence, Choudhury et al. (2004) developed a nonlinearity index by comparing the maximum squared bicoherence with the average squared bicoherence plus two times the standard deviation of the estimated squared bicoherence. The disadvantage of using this index is that the presence of a few large peaks significantly bias the standard deviation and mean of the estimated bicoherence, which leads to some false negatives. The other problem with this index is that it cannot give an indication of the extent 
of nonlinearity because with the increase of peak sizes, the mean and the standard deviation also increase. In order to avoid these limitations, the index can be modified as:

$$
\left.N L I_{\text {modified }} \triangleq b \hat{i c}^{2} \max -\overline{\left(\hat{b i c}_{\text {robust }}^{2}\right.}+2 \sigma_{b_{\hat{i c}}{ }^{2}, \text { robust }}\right)
$$

where $b i c_{\text {robust }}^{2}$ and $\alpha_{\text {bic }^{2} \text {,robust }}$ are the robust mean and the robust standard deviation of the estimated squared bicoherence. They are calculated by excluding the largest and smallest $Q \%$ of the bicoherence. A good value of $Q$ may be chosen as 10 . There is a similar concept used in statistics to calculate robust mean called 'trimmed mean'. The users may choose a different value of $Q$.

Therefore, it can be concluded that:

- if $N L I_{\text {modified }} \leq \cdot 0$, the signal generating process is LINEAR

- if $N L I_{\text {modified }}>0$, the signal generating process is NONLINEAR

Since the squared bicoherence is bounded between 0 and 1 , the Nonlinearity Index $(N L I)$ is also bounded between -1 and 1 .

\subsubsection{Total Nonlinearity Index (TNLI)}

It is important to measure nonlinearity in terms of a metric especially when there is a need to compare the extent of nonlinearities in various time series. If a time series is detected as nonlinear by the above-mentioned $N G I_{\text {modified }}$ and $N L I_{\text {modified }}$ indices, then the total nonlinearity present in the time series can be quantified using the following new index:

$$
T N L I \triangleq \sum b i c_{\text {significant }}^{2}
$$

where, TNLI is Total Nonlinearity Index, bic $^{2}$ significant are those bicoherence which fail the hypothesis test in Equation (8), i.e., $2 K \operatorname{bic}^{2}\left(f_{1}, f_{2}\right)>c_{\alpha}^{\chi^{2}}$. The TNLI is bounded between 0 and $L$, where $L$ is the number of bic $_{\text {significant. }}^{2}$

\subsubsection{Illustrative Example 1}

The purpose of this example is to demonstrate the efficacy of the proposed indices in the presence of varying noise and extent of nonlinearity of a signal. Let a signal be generated as follows:

$$
\begin{aligned}
& x^{\prime}(k)=\sin \left(2 \pi f_{1} k+\phi_{1}\right)+\sin \left(2 \pi f_{2} k+\phi_{2}\right) \\
& x(k)=x^{\prime}(k)+d(k) \\
& y(k)=x^{\prime}(k)+n_{l} x^{\prime}(k)^{2}+d(k)
\end{aligned}
$$

where, $f_{1}=0.12, f_{2}=0.30, \varphi_{1}=\pi / 3, \varphi_{2}=\pi / 8, n_{l}$ is a multiplication factor employed to represent the contribution of the nonlinear component of the signal, and $d(k)$ is a white noise sequence. Again, frequencies are normalized such that the sampling frequency is 1 . The quadratic term in Equation 16 will introduce phase coupled nonlinearity in the output signal, $y$. The quadratically phase coupled nonlinearities arise due to the interactions between any two of the signal components at frequencies $f^{1}, f_{2}, 2 f_{1}, 2 f_{2}, f_{2}-f_{1}$, and $f_{1}$ $+f_{2}$. For details refer to (Choudhury, 2004).

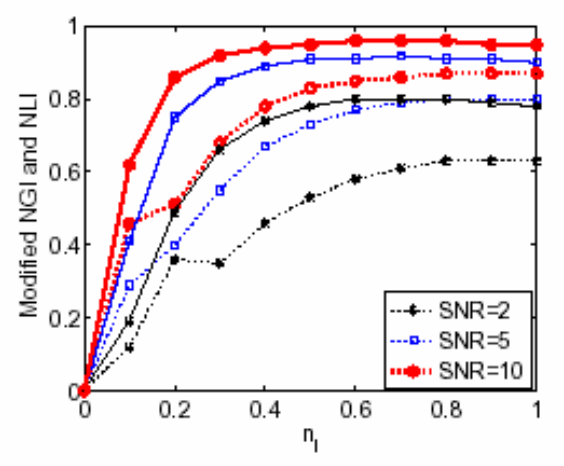

Fig. 2. $N G I_{\text {modified }}$ (dotted lines) and $N L I_{\text {modified }}$ (solid lines).

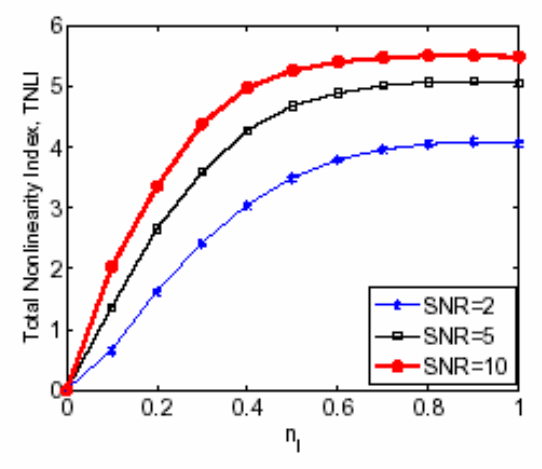

Fig. 3. TNLI increases with the increase of the nonlinearity of the signal.

Figure 2 shows the modified Non-Gaussianity Index $\left(N G I_{\text {modified }}\right)$ and the modified Nonlinearity Index $\left(N L I_{\text {modified }}\right)$ plotted against $n l$ for varying cases of signal to noise $(S N R)$ ratio. The dotted lines represent the modified NGI and the solid lines show the modified NLI. It is clear from the figure that both 
indices increase with the increase of nonlinearity in the signal. The total nonlinearity index shown in Figure 3 represents the extent of nonlinearity in the signal. For $n_{l}=0$, there is no nonlinearity. Therefore, $T N L I=0$. With increase of $n_{l}$, the TNLI also increases. The amount of noise influences the calculation of all indices because the denominator of the bicoherence is affected by the noise.

\section{Investigating nonlinearity of a Continuously Stirred Tank Reactor (CSTR)}

In this section, the nonlinearity of a non-isothermal continuously stirred tank reactor (CSTR) is measured around the operating point used for Case 1 in Marlin (1995).The schematic of the CSTR process is shown in Figure 4.

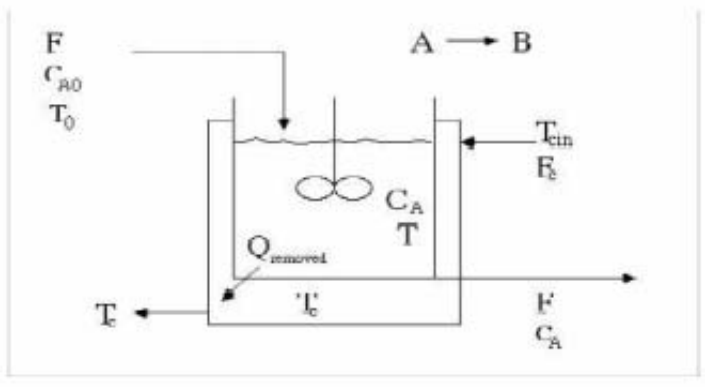

Fig. 4. Schematic of the CSTR

A simple first order irreversible exothermic reaction of the form $A \rightarrow B$ is considered here. Assuming a perfect mixing in the reactor and the jacket, the CSTR model can be described by the following differential equations:

$$
\begin{aligned}
& \frac{d c_{A}}{d t}=\frac{F}{V}\left(c_{A 0}-c_{A}\right)-k_{0} e^{-E / R T_{C_{A}}} \\
& \frac{d T}{d t}=\frac{F}{V}\left(T_{0}-T\right)-\frac{a F_{c}^{b+1}}{V \rho C_{P}\left(F_{c}+\frac{a F_{c}^{b}}{2 \rho_{c} C_{p c}}\right)}\left(T-T_{c l n}\right) \\
& +\frac{1}{\rho C_{p}}\left(-\Delta H_{r \times n}\right) k_{0} e^{-E / R T_{C_{A}}}
\end{aligned}
$$

The notation and the parameters for simulation are given in Table 1. The simulation of the CSTR with the above mentioned parameters results in a steady state value of $0.2645 \mathrm{kmole}=m 3$ for $C A$ and $393.9 \mathrm{~K}$ for the reactor temperature, $T$. Sinusoids with varying amplitudes and frequencies were used to excite the process and the subsequent nonlinearity was quantified for each case. The results are shown in Figure 5. The figure clearly shows that total nonlinearity increases with the increase of the amplitude of the excitation signal. Also, an increase in frequency of the input excitation causes an increase in nonlinearity.

\begin{tabular}{|c|c|c|c|}
\hline Symbol & Description & magnituds & unit \\
\hline F & Reactant flow-rate & 1 & $m^{3 / m i n}$ \\
\hline$C_{A 0}$ & initial conc. of $\mathrm{A}$ & 2 & kmole $\mathrm{A} / \mathrm{m}^{3}$ \\
\hline $\mathrm{V}$ & Reactor Volume & 1 & $m^{3}$ \\
\hline$C_{p}$ & cutlet str. sp. he at & 1 & $\mathrm{cal} / \mathrm{g}^{\circ} \mathrm{C}$ \\
\hline$\rho$ & cutlet str. density & $10^{5}$ & $g / m^{3}$ \\
\hline$T_{o}$ & Foed temp. & 323 & K \\
\hline$C_{p c}$ & Coolant sp. beat & 1 & $\mathrm{cal} / \mathrm{g}^{\circ} \mathrm{C}$ \\
\hline$F_{c}$ & Coolant flow rate & 15 & $m^{3} / \min$ \\
\hline$T_{\text {cin }}$ & Coolant Inkt temp. & 365 & $K$ \\
\hline$\rho_{c}$ & density of coolant & $10^{5}$ & $g / m^{3}$ \\
\hline$-\Delta H_{r \times n}$ & Heat of reaction & $1.3 \times 10^{3}$ & calkmole A \\
\hline$k_{0}$ & constant & $1.0 \times 10^{10}$ & $\min ^{-1}$ \\
\hline E/R & constant & 8330.1 & $K^{-1}$ \\
\hline $\mathrm{b}$ & constant & 0.5 & $K^{-1}$ \\
\hline $\mathrm{a}$ & constant & $1.68 \times 10^{6}$ & $K^{-1}$ \\
\hline
\end{tabular}

Table 1. CSTR parameters for the first order reaction

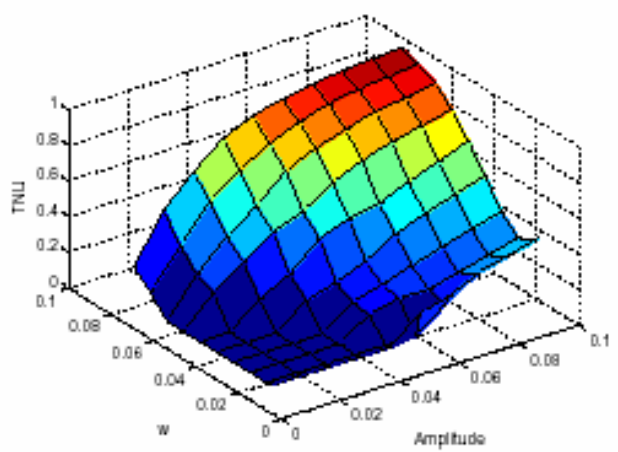

Fig. 5. Dependency of CSTR nonlinearity on the input signal's amplitude and frequency

\section{Industrial Case Study 1 - Eastman Chemical Plant}

An industrial data set was provided by the Advance Controls Technology group of Eastman Chemical Company. Figure 6 shows the process schematic of the plant, which contains three distillation columns, two decanters and several recycle streams. There are 15 control loops and 15 indicators on the schematic. There are eight flow controllers. Six of them are in cascade configuration. The Advanced Controls Technology group had identified a need for diagnosis of a common disturbance with an oscillation period of about 2 hours. Thornhill et al. (2003b) had found out and confirmed that the root cause was a control valve problem. In this section, the newly proposed procedure is applied to this data set to demonstrate its efficacy in the detection and diagnosis of the plant-wide disturbance. 


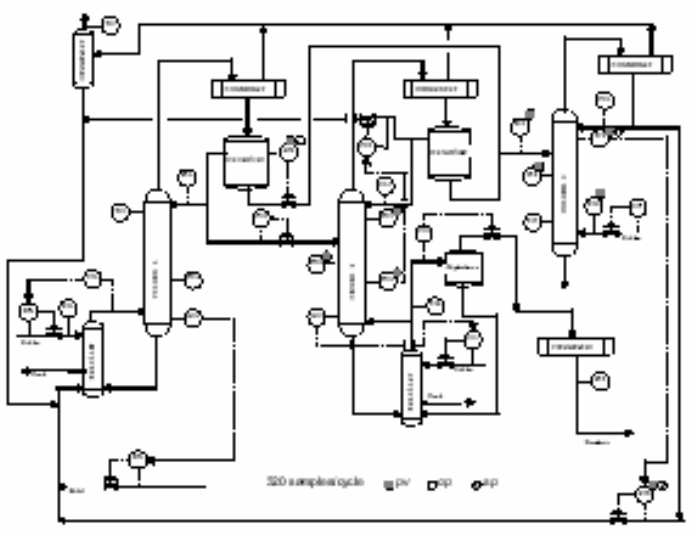

Fig. 6. Process schematic. The main oscillation variables are marked by circle symbols.

\subsection{Data Description}

Uncompressed plant data were collected at a sampling period of $20 \mathrm{~s}$ for the period of two days. Therefore, there are 8640 samples for each tag. The data set contains 48 variables: 14 process variables ( $p v$ 's), 14 controller outputs (op's), 15 indicator variables and 5 cascade loop setpoints ( $s p$ 's).

\subsection{High Density Plots}

Figure 7 shows the time trends and power spectra of the first 15 variables which are $p v$ 's.
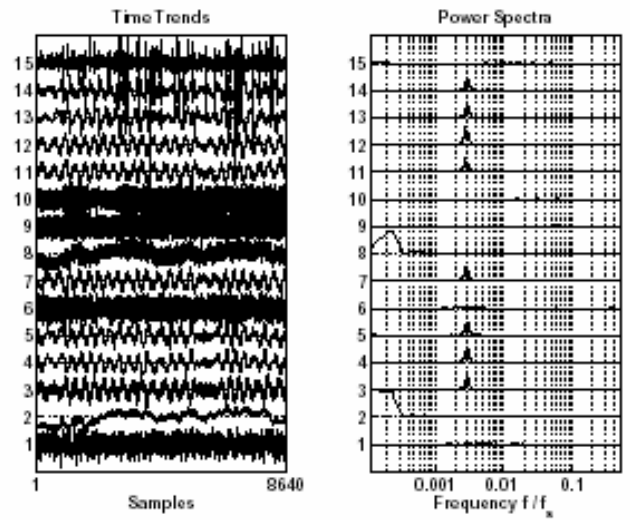

Fig. 7. Time trend and power spectra of $15 \mathrm{pv}$ 's

The power spectra shows the presence of oscillation at the frequency of 0.003 cycles/sample (or about 333 samples/cycle, nearly a period of 2 hours). This oscillation had propagated through out the adjacent units and affected many variables in the process. Figure 8 show the time trends of controller output signals and the indicator variables with their spectra, respectively. These figures also show that there is a dominant oscillation with frequency 0.003 cycles/sample which affects many of the variables.
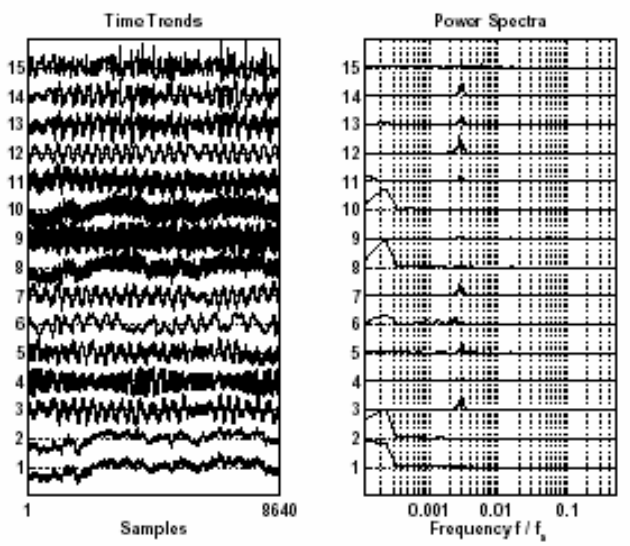

Fig. 8. Time trend and power spectra of $15 \mathrm{op}$ 's

\subsection{Reduction of the problem size}

Thornhill et al. (2003b) performed nonlinearity analysis using surrogate data method on all 48 variables. In order to troubleshoot an oscillation, it is better to investigate the control error signals for the possible presence of oscillations in them. Figure 9 shows the time trends and power spectra of the control error signal for the 15 control loops. This figure clearly shows that only four loops namely LC1, TC1, LC2 and TC2 have oscillations at a frequency of 0.003 cycles/sample (or about 333 samples/cycle, nearly a period of 2 hours). Therefore, as a first attempt to diagnose the root-cause of this oscillation, only these four control loops were examined for performing a nonlinearity test.
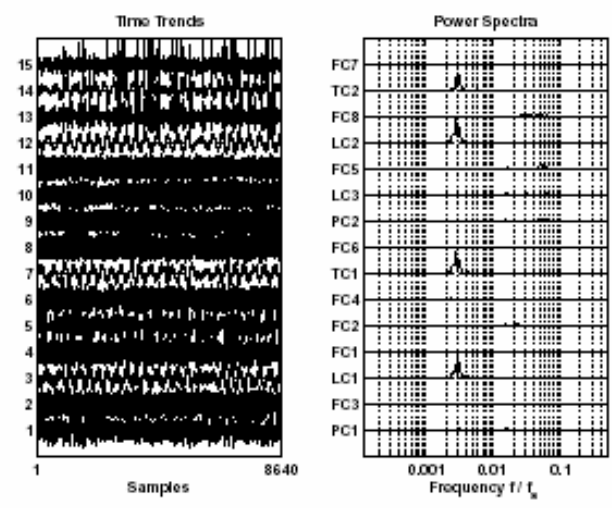

Fig. 9. Time trend and power spectra of 15 error signals 


\subsection{Detection of Plantwide Oscillation by PSCMAP}

Figure 10 shows the PSCMAP of the 15 error variables. It is again clear from this figure that only LC1, TC1, LC2 and TC2 loops have similar oscillation.

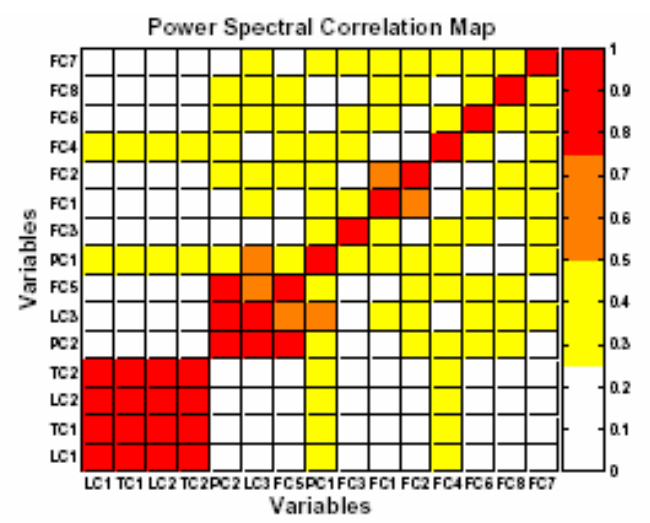

Fig. 10. Power Spectral Correlation Map (PSCMAP) for 15 error signals

\subsection{Nonlinearity analysis using bicoherence based indices}

Nonlinearity analysis has been performed on the error signals of the four control loops. In order to exclude any effect from oscillations other than the frequency of 0.003 cycles/sample (or about 333 samples/cycle, nearly a period of 2 hours), the data was filtered using a band-pass Wiener filter with boundaries of [0.001 to $0.1]$ cycles/sample. Then, the filtered data were downsampled by a factor of 10 in order to make them suitable to use with the parameters of the bicoherence estimation algorithm. The direct method of bicoherence estimation using an approach similar to Welch's average periodogram estimation was used where a 128 point FFT was implemented using 128 samples for each data segment. For details, refer to (Choudhury, 2004). Downsampling the data by a factor of 10 reduces the oscillation period to approximately 34 samples/cycle. Therefore, a 128 samples segment of the data contains enough number of cycles of oscillation to perform a bicoherence estimation. The results of this analysis are shown in Table 2.

Table 2. Nonlinearity analysis results for the Eastman Chemical Plant data

\begin{tabular}{|c|c|c|c|}
\hline \hline Tag Name & NGI & NLI & TNLI \\
\hline LC1 & 0.048 & 0.3 & 0.297 \\
\hline TC1 & 0.178 & 0.43 & 0.428 \\
\hline LC2 & 0.202 & 0.57 & 0.902 \\
\hline PC2 & 0.068 & 0.32 & 0.636 \\
\hline \hline
\end{tabular}

The table clearly shows that TNLI is highest for loop LC2, i.e., this loop has the maximum nonlinearity. This is also the loop that was identified as a root-cause in a previous analysis performed by (Thornhill et al., $2003 \mathrm{~b}$ ). Therefore, it can be concluded that the Total Nonlinearity Index (TNLI) can correctly identify the root cause of plantwide Oscillation problems caused by nonlinearity related faults.

\subsection{Diagnosis of the problem in Loop LC2}

Once a nonlinearity is detected using higher order statistical method, the $p v$-op plot is used to diagnose its cause. It is well known (Hagglund, 1995; Rengaswamy et al., 2001; Choudhury et al., 2005; Choudhury, 2004) that the presence of stiction in control valve in a control loop produces limit cycles in the controlled variable $(p v)$ and the controller output $(o p)$. For such a case, the $p v-o p$ plot shows elliptical cyclic patterns, which are taken as a signature of valve stiction. If no such patterns are observed, it is concluded that there may be valve problems other than stiction. Note that for the cases of tightly tuned controller or a process with time delay, the $p v-o p$ plot may also exhibit elliptical patterns. But they do not add nonlinearity in a control loop. Therefore, these cases do not pass the nonlinearity test. The $p v-o p$ plot is investigated only after a successful nonlinearity detection in the loop. That is why the $p v$-op plot should not be used alone to detect stiction. This must be used in conjunction with the nonlinearity test. For a detailed discussion on these issues, refer to Choudhury et al. (2004), Choudhury et al. (2006).

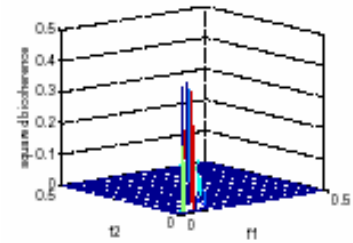

(a) Bicoherence Plot

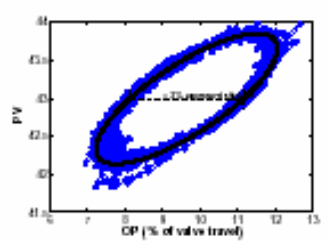

(b) LC2.pv- LC2.op plot
Fig. 11. Oscillation diagnosis plots for LC2 loop

Figure 11 shows the diagnostic plots for this loop. Figure 11(a) shows that there are significantly large peaks in the bicoherence plot indicating a nonlinear loop. The values of $N G I$ and $N L I$ for this loop are 0.15 and 0.42 , respectively, which clearly indicates that the loop exhibits nonlinearity. Once a loop nonlinearity is detected, it should be checked whether this is due to stiction or other process nonlinearity. Figure 11(b) shows the $p v$-op plot for this loop. The plot clearly shows an elliptic pattern indicating the presence of stiction in the control valve. The apparent stiction is 
quantified to be approximately $3 \%$ using the method described in Choudhury et al. (2006).

Similar results of root cause diagnosis were also discussed in Thornhill et al. (2003b). It was reported that the control valve of loop LC2 suffered from a high static friction or stiction problem (Thornhill et al., $2003 b$ ). It has been confirmed that the control valve caused control variable LC2.pv to oscillate, and the oscillation passed through the feedback controller and made the controller output LC.op also to oscillate. After that, the oscillations propagated to the temperature control loop TC1 in the second distillation column and caused the temperature to oscillate. This is the reason why temperature indicator TI4.pv and control variable TC1.pv had oscillations too. For more information, refer to Thornhill et al. (2003b).

\section{Industrial Case Study 2 - SE Asia Refinery}

This case study describes the method applied to a refinery data set, courtesy of a SE Asian Refinery. A simplified schematic of the refinery process is shown in Fig 12.

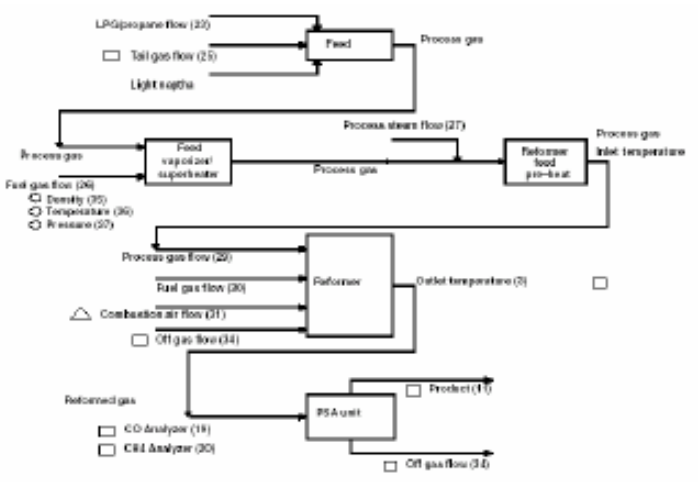

Fig. 12. Schematic of the SEA Refinery Process

The data set consisting of 512 samples of 37 measurements sampled at 1 min interval. It comprises measurements of temperature, flow, pressure and level loop along with some composition measurements from the gas analyzers. The process contains a recycle loop from the PSA unit to the reformer unit. Controller errors $(S P P V)$ are analyzed for control loop measurements. The time trends of the controller errors and their corresponding power spectrum are already presented in Figure 1. It is clear from both figures that there is very dominant oscillation at the frequency of 0.06 or approximately 17 samples/cycle. This particular oscillation was all over the plant and affected the plant operation a lot.

\subsection{Oscillation Detection by PSCMAP}

The rearranged spectral correlation colour maps is shown in Figure 13. This clearly identifies that the tags $2,3,4,8,9,10,11,13,15,16,19,20,24,25,28,33$ and 34 have similar spectral shape and are affected with the above-mentioned oscillation.

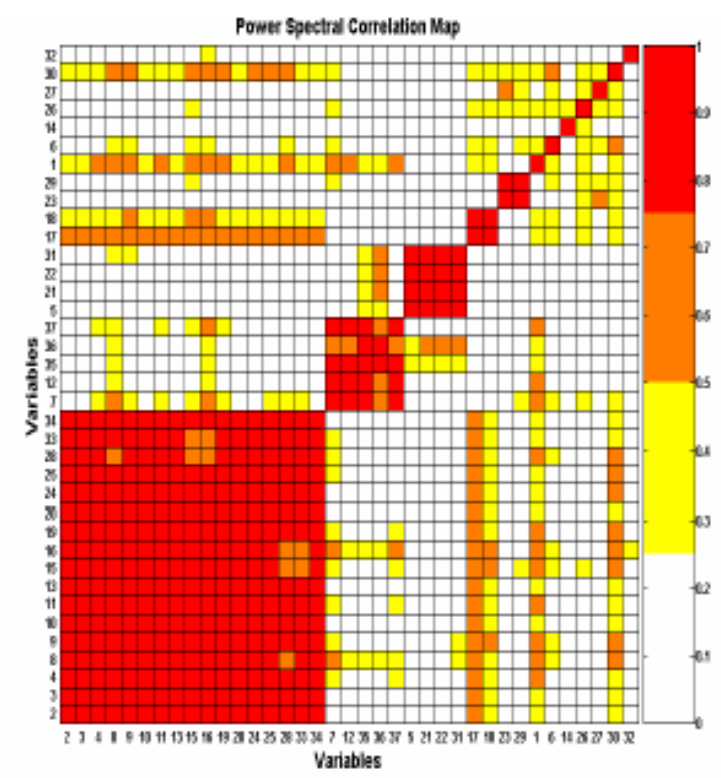

Fig. 13. Schematic of the SEA Refinery Process

\subsection{Oscillation Diagnosis}

The power spectral correlation map finds that tags 2,3 , $4,8,9,10,11,13,15,16,19,20,24,25,28,33$ and 34 are oscillating with the frequency 0.06 . The total nonlinearity test was applied to only these tags to trou-

Table 3. Nonlinearity analysis results for SEA Refinery data set

\begin{tabular}{|c|c|c|c|}
\hline \hline Tag Name & NGI & NLI & TNLI \\
\hline 2 & 0.15 & 0.99 & 2.71 \\
\hline 3 & 0.14 & 0.94 & 2.65 \\
\hline 4 & 0.06 & 0.81 & 0.81 \\
\hline 8 & 0.06 & 0 & 0 \\
\hline 9 & 0 & 0 & 0 \\
\hline 10 & 0.04 & 0.79 & 0.79 \\
\hline 11 & 0.20 & 0.96 & 2.84 \\
\hline 13 & 0.15 & 0.96 & 1.80 \\
\hline 15 & 0.05 & 0.80 & 0.80 \\
\hline 16 & 0.09 & 0.49 & 1.68 \\
\hline 19 & 0.13 & 0.88 & 0.88 \\
\hline 20 & 0.13 & 0.94 & 1.76 \\
\hline 24 & 0.01 & 0.76 & 0.76 \\
\hline 25 & 0 & 0 & 0 \\
\hline 28 & 0.01 & 0.76 & 0.76 \\
\hline 33 & 0.08 & 0.87 & 3.32 \\
\hline 34 & 0.20 & 0.99 & 7.63 \\
\hline \hline
\end{tabular}


-bleshoot this oscillation. Table 3 shows the results of this analysis. It is clear from the figure that the tag 34 is the most nonlinear tag because the total nonlinearity index (TNLI) is highest for this tag. Therefore, the tag 34 can be treated as the potential root-cause of this plantwide oscillation problem. This finding is in agreement with other previous studies performed on this data set (Thornhill et al., 2001).

\section{Conclusions}

This paper presents a systematic method to diagnose the root-cause of a plant-wide oscillation problem. The plant-wide oscillation can be detected using high density plot or spectral correlation color map. Then the variables oscillating together are tested for possible presence of nonlinearity in them. The variable or the control loop that has the highest amount of nonlinearity should be treated first as a possible candidate for the cause of the plant-wide oscillation. A list for priority maintenance can be prepared based on the Total Nonlinearity Index (TNLI). The $p v$-op plot can be used to diagnose the fault of a control loop. The proposed method has successfully been applied on simulated as well as industrial data sets.

\section{Acknowledgements}

The author gratefully acknowledges Eastman Chemical Company and SE Asia Refinery for their help and permission to publish the results.

\section{References}

1. Bialkowski,W. L. (1992). Dreams vs. reality: A view from both sides of the gap. In: Control Systems. Whistler, BC, Canada. pp. 283-294.

2. Choudhury, M. A. A. S. (2004). Detection and Diagnosis of Control Loop Nonlinearities, Valve Stiction and Data Compression. PhD thesis.

3. Choudhury, M. A. A. S., N. F. Thornhill and S. L. Shah (2005). Modelling valve stiction. Control Engineering Practice 13, 641-658.

4. Choudhury, M. A. A. S., S. L. Shah and N. F. Thornhill (2004). Diagnosis of poor control loop performance using higher order statistics. Automatica 40(10), 1719-1728.

5. Choudhury, M. A. A. S., S. L. Shah, N. F. Thornhill and D. Shook (2006). Automatic detection and quantification of stiction in control valves. Control Engineering Practice 14, 13951412.

6. Collis, W.B., P.R. White and J.K. Hammond (1998). Higher-order spectra: The bispectrum and trispectrum. Mechanical Systems and Signal Processing 12, 375-394.
7. Desborough, L. and R. Miller (2002). Increasing customer value of industrial control performance monitoring - honeywell's experience. In: Preprint of Chemical Process Control, CPC-6. Tucson, Arizona. pp. 153-186.

8. Hagglund, T. (1995). A control loop performance monitor. Control Engg. Practice 3(11), 15431551.

9. Helbig, A., W. Marquardt and F. Allg"ower (2000). Nonlinearity measure: definition, computation and applications. Journal of Process Control 10, 113123.

10. Hinich, M. J. (1982). Testing for gaussianity and linearity of a stationary time series. Journal of time series analysis 3, 169-176.

11. Jiang, H., M.A.A.S. Choudhury, S.L. Shah, J.W. Cox and M. A. Paulonis (2006). Detection and diagnosis of plant-wide oscillations via the spectral envelope method. In: In the proceedings of ADCHEM 2006. Gramado, Brazil. pp. 11391144.

12. Marlin, T. E. (1995). Process Control: Designing Processes and Control Systems for Dynamic Performance. McGraw Hill. New York.

13. Martin, G. D., L.E. Turpin and R. P. Cline (1991). Estimating control function benefits. Hydrocarbon Processing pp. 68-73.

14. Miao, T. and D. E. Seborg (1999). Automatic detection of excessively oscillatory control loops. In: Proceedings of the 1999 IEEE International Conference on Control Applications. Kohala Coast-Island of Hawai'i, Hawai'i, USA.

15. Nikias, C. L. and A. P. Petropulu (1993). HigherOrder Spectra: A nonlinear signal processing frame work. Prentice Hall. New Jeresy.

16. Rao, T. S. and M. M. Gabr (1980). A test for linearity and stationarity of time series. Journal of time series analysis 1(1), 145-158.

17. Rengaswamy, R., T. Hagglund and V. Venkatasubramanian (2001). A qualitative shape analysis formalism for monitoring control loop performance.. Engng. Appl. Artificial Intell. 14, 23-33.

18. Shunta, J. P. (1995). Achieving world class manufacturing through process control. PrenticeHall. NJ, USA.

19. Taha, O., G. A. Dumont and M. S. Davies (1996). Detection and diagnosis of oscillations in control loops. In: Proceedings of the 35th conference on Decision and Control. Kobe, Japan.

20. Tangirala, A.K., S.L. Shah and N.F. Thornhill (2005). Pscmap: A new tool for plant-wide oscillation detection. Journal of Process Control 15, 931941.

21. Thornhill, N. F., B. Huang and H. Zhang (2003a). Detection of multiple oscillations in control loops. Journal of Process Control 13, 91-100. 
22. Thornhill, N. F., F. Cox and M. Paulonis (2003b). Diagnosis of plant-wide oscillation through datadriven analysis and process understanding. Control Engng. Prac. 11(12), 1481-1490.

23. Thornhill, N. F., S. L. Shah and B. Huang (2001). Detection of distributed oscillations and rootcause diagnosis. In: Preprints of CHEMFAS-4 IFAC. Korea. pp. 167-172.

24. Thornhill, N. F., S. L. Shah, B. Huang and A. Vishnubhalota (2002). Spectral principal component analysis of dynamic process data. Control Engng. Prac. 10, 833-846.

25. Zang, X. and J. Howell (2005). Isolating the root cause of propagated oscillations in process plants.
International Journal of Adaptive Control Signal Processing 19, 247-265.

26. Zang, X. and J. Howell (2006). Isolating the source of whole-plant oscillations through biamplitude ratio analysis. Control Engineering Practice, 2005, In Press, corrected proof, available online 5 June 2006. 